\title{
Photometrischer MIR-ATR-Sensor für die Prozessanalyse
}

\author{
Daniel Geörg ${ }^{1}$, Matthias Gornicki ${ }^{1}$, Dominik Egly ${ }^{1}$, Frank-Jürgen Methner ${ }^{2}$, Thomas Beuermann ${ }^{1}$ \\ ${ }^{1}$ Hochschule Mannheim, Institut PI, Paul-Wittsack-Str. 10, 68163 Mannheim, Deutschland, \\ d.geoerg@hs-mannheim.de \\ ${ }^{2}$ TU Berlin, Fachgebiet Brauwesen -GG4-, Seestr. 13, 13353 Berlin, Deutschland
}

\begin{abstract}
There is a strong demand for on-line monitoring in modern process automation. Transferring analytical methods from laboratory to production plants is still a problem, since for many laboratory devices do not exist corresponding process-capable measuring instruments. The developed MIR-ATR sensor solves this problem in case of FT-MIR spectroscopy (wavelength range $2.5 \mu \mathrm{m}$ to $25 \mu \mathrm{m}$ or wavenumber range $4000 \mathrm{~cm}^{-1}$ to $400 \mathrm{~cm}^{-1}$ ). A prototype of this sensor was constructed in order to determine the alcohol and sugar concentration in the brewing and beverage industry. Furthermore, the consumption of carbon source sugar can be monitored during yeast fermentations. The sensor can easily be adapted to a wide range of chemical, pharmaceutical and biotechnological processes by exchanging the analyte specific optical band pass filters.
\end{abstract}

Key words: optical sensor, MIR-ATR sensor, in-line sensor, ethanol sensor, sugar sensor

\section{Einleitung}

Moderne industrielle Prozessautomatisierung erfordert Online-Messtechnik. Jedoch sind für einen großen Teil etablierter Labormessgeräte keine prozessfähigen Pendants verfügbar, sodass nach einer analytischen Prozessbegleitung im Labor große Hürden für die Übertagung der Messmethode in den Technikums- oder Produktionsmaßstab bestehen. Der entwickelte MIR-ATR-Sensor schließt diese Lücke für die im Labor häufig zur Konzentrationsbestimmung verwendete FTMIR-Spektroskopie mit mittelinfrarotem Licht (Wellenlängenbereich $2,5 \mu \mathrm{m}$ bis $25 \mu \mathrm{m}$ bzw. Wellenzahlbereich $4000 \mathrm{~cm}^{-1}$ bis $400 \mathrm{~cm}^{-1}$ ). Ein Prototyp des MIR-ATR-Sensors wurde im Hinblick auf Anwendungen in der Getränkeindustrie und die Prozessverfolgung von HefeFermentationen so ausgelegt, dass mit inm die Analyten Zucker und Ethanol bestimmt werden können. Um andere Analyten nachzuweisen, müssen lediglich entsprechende optische Bandpassfilter ausgewählt und eingebaut werden. Somit kann der Sensor bei einer Vielzahl chemischer, pharmazeutischer und biotechnologischer Prozesse eingesetzt werden.

In der Produktion, insbesondere in der Getränkeindustrie, werden häufig zur Prozessüberwachung Sensoren eingesetzt, welche lediglich Summenparameter wie Schallgeschwindigkeit, Brechungsindex, Trübung, o.ä. bestimmen und nicht spezifisch für einen
Analyten sind. Dadurch können bei Prozessvariationen leicht Querempfindlichkeiten zwischen den einzelnen Messparametern auftreten.

Im Labor werden bereits seit einigen Jahren FT-MIR-Spektrometer für die Online-Prozesskontrolle von Fermentationen eingesetzt [1] [2] [3]. Dieser Messaufbau ermöglicht die gleichzeitige Bestimmung der Konzentration einer Vielzahl von Stoffen ohne Zeitverzug. Durch den sensiblen Aufbau der Optik mit bewegten Teilen kann ein FT-MIR-Spektrometer jedoch nur mit hohem Aufwand für die Produktion in ein robustes, prozessfähiges Messgerät umgerüstet werden. Dabei muss das Spektrometer häufig in ein ex-sicheres und staub- bzw. wassergeschütztes Gehäuse eingebaut werden. Die Kosten für eine Prozessimplementierung liegen dadurch leicht in der Größenordnung von $100.000 € \mathrm{Im}$ Gegensatz zum MIR-Bereich gibt es im UV/VIS- und NIR-Bereich faseroptische Lichtleiter, mit welchen größere Distanzen zwischen Messsonde und Spektrometer überbrückt werden können, sodass lediglich die Sonde in der Produktionsanlage bzw. am Reaktor zu installieren ist und das Spektrometer z.B. in der geschützten Messwarte untergebracht werden kann. Jedoch weisen viele Moleküle im UV/VIS- und NIRBereich keine spezifischen Banden auf, was eine quantitative Bestimmung in komplexen Mischungen sehr erschwert. Der entwickelte robuste und kompakte MIR-ATR-Sensor bringt 
die hohe Spezifität des MIR-Bereichs mit der geforderten Prozesstauglichkeit in Einklang, indem er direkt an den Messprozess adaptiert bzw. angeflanscht werden kann. Bei der eingesetzten ATR-Technik werden die Proben in abgeschwächter Totalreflexion vermessen, um die im MIR-Bereich erforderlichen geringen Schichtdicken von wenigen Mikrometern zu realisieren.

\section{ATR-Messmethode}

Elektromagnetische Strahlung wie UV/VIS-, NIR- und MIR-Licht kann durch Moleküle absorbiert werden. Dabei ist die spektrale Lage und Form der Absorptionsbanden charakteristisch für die jeweilige chemische Verbindung. Daher ist es möglich, Stoffe über die spektrale Lage der Absorptionsbanden zu identifizieren und deren Konzentration durch Messen der Absorption $A(\lambda)$ zu bestimmen. Es gilt das Lambert-Beersche Gesetz [4]:

$$
A(\lambda)=\log _{10}\left(\frac{I_{0}(\lambda)}{I_{1}(\lambda)}\right)=\varepsilon(\lambda) \cdot c \cdot d
$$

mit $\lambda$ : Wellenlänge des Anregungslichts, $I_{0}$ : Intensität bei der Bezugsmessung, $l_{1}$ : Intensität bei der Probenmessung, $c$ : Stoffkonzentration, $\varepsilon(\lambda)$ : stoffspezifischer molarer dekadischer Extinktionskoeffizient, $d$ : optische Weglänge.

In der Regel ist das Lambert-Beersche Gesetz (Linearität) nur für Absorptionen kleiner 2 gültig. Da im mittelinfraroten Spektralbereich die Matrix bei flüssigen Proben (z.B. Wasser oder Lösungsmittel) selbst absorbiert, muss in obiger Gleichung die optische Schichtdicke $d$ in der Größenordnung von wenigen Mikrometern liegen, um entsprechend kleine Absorptionswerte zu erhalten. Eine Möglichkeit dies technisch umzusetzen, ist die ATR-Messmethode (ATR: abgeschwächte Totalreflexion), bei welcher das einfallende Licht an einer Grenzfläche zur Probe total reflektiert wird [5]. Die Skizze in Abbildung 1 zeigt schematisch den optischen Strahlengang im entwickelten MIR-ATR-Sensor. Das einfallende Licht wird an der Grenzfläche ATR-Prisma/Probe total reflektiert und dringt dabei weniger als eine halbe Wellenlänge in die Probe ein. Dadurch werden im MIR-Bereich (Wellenlänge: 2,5 $\mu \mathrm{m}$ bis $25 \mu \mathrm{m}$ ) optische Schichtdicken von wenigen Mikrometern realisiert. Das innerhalb der Eindringtiefe nicht absorbierte Licht wird total reflektiert und gelangt zum spektralen Nachweis auf den Detektor. Je nach Probe und ATR-Aufbau (Material des optischen Kristalls, Einfallswinkel, Anzahl der Reflexionen an der Grenzfläche) liegen die gemessenen Absorptionen im Bereich von 0,001 bis 1.

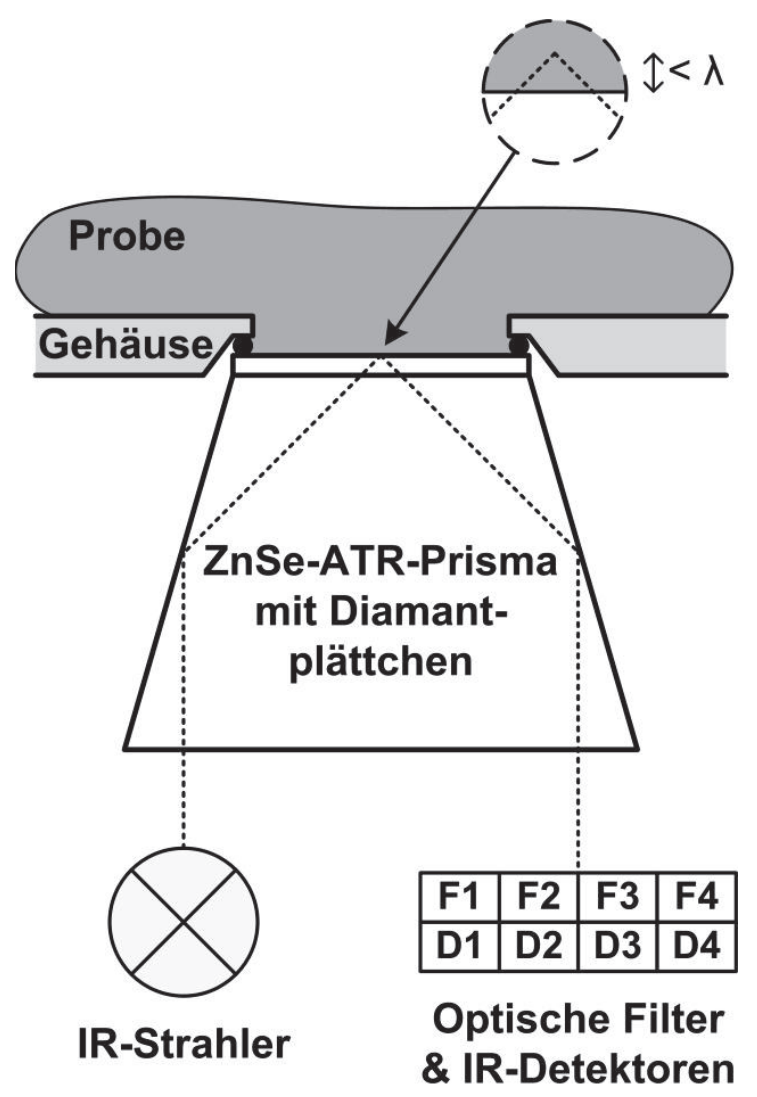

Abb. 1: MIR-ATR-Sensor mit einer Reflexionsstelle.

\begin{abstract}
Aufbau des Sensors
Abbildung 2a zeigt den CAD-Entwurf des Sensors. Die optischen Bandpassfilter sind im Deckel des Detektorgehäuses integriert. So ist es durch Austausch des 4-Kanal-Detektors möglich, den Sensor für andere Anwendungen umzurüsten, da das spektrale Ansprechverhalten lediglich durch die optischen Filter festgelegt wird. Die IR-Strahlung wird thermisch detektiert, d.h. die Erwärmung der Detektorfläche durch die einfallende Strahlung wird in ein elektrisches Signal umgesetzt. Dieses Detektionsprinzip ist preisgünstig und die spektrale Empfindlichkeit konstant, was das Umrüsten auf verschiedene Messanwendungen erleichtert. Die Lichtquelle ist ein elektrisch modulierter Temperaturstrahler, so dass ein in vielen Photometern eingesetzter mechanischer Chopper entfällt. Im Vergleich zu einem FTMIR-Spektrometer benötigt das Photometer insbesondere kein Interferometer mehr, so dass der Sensor keine beweglichen Teile enthält.
\end{abstract}



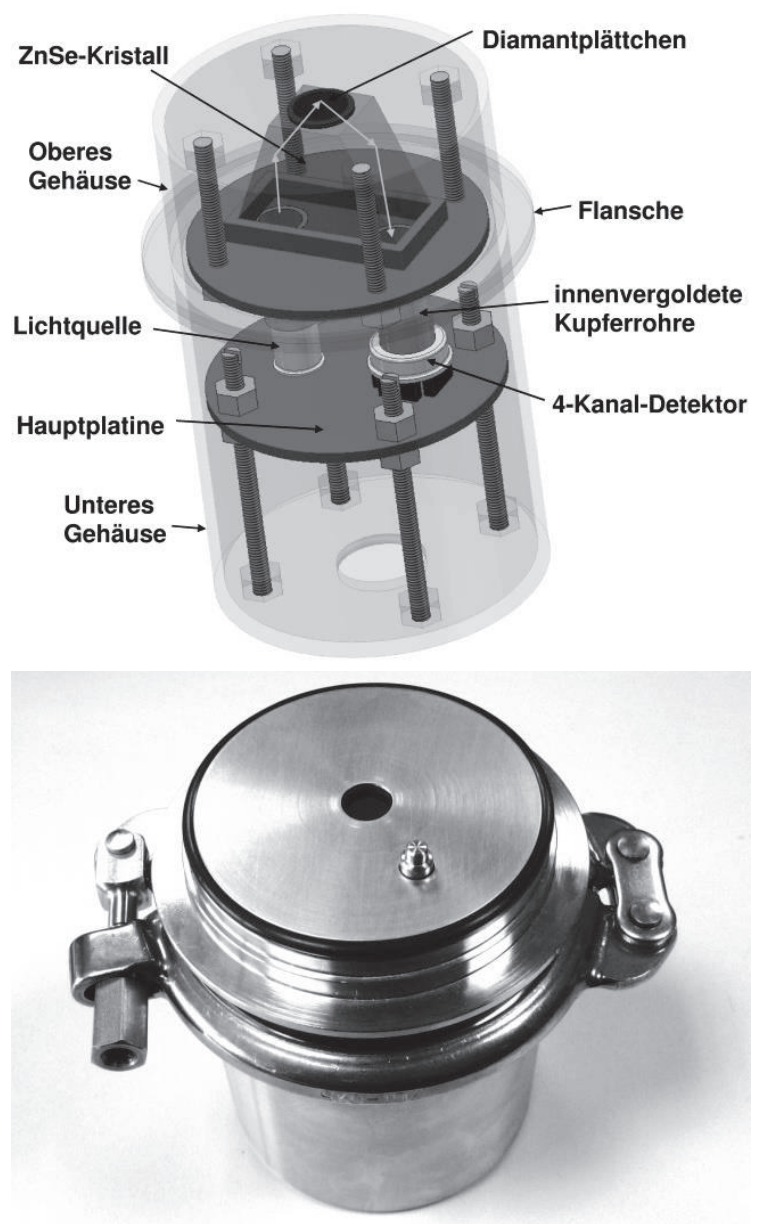

Abb. 2: a) CAD-Entwurf des MIR-ATR-Sensors, b) erster Prototyp mit Varivent ${ }^{\circledR}$-Prozessflansch.

Die IR-Strahlen werden mittels Kupferrohre auf die ATR-Optik gerichtet. Diese besteht aus einem ZnSe-Prisma zur Strahlführung und einem chemisch inerten und mechanisch robusten Diamantplättchen an der ATRGrenzfläche zur Probe bzw. zum Prozess.

Abbildung $2 \mathrm{~b}$ zeigt ein Foto des ersten Prototyps. Die Oberfläche des ATR-Prismas ist in einen Varivent ${ }^{\circledR}$-Prozessflansch integriert. Dies ermöglicht den Einsatz in hygienischen Prozessen und zeigt die kompakte Bauweise des Sensors.

\section{Wahl der optischen Filter}

In einem Photometer werden keine kompletten Spektren aufgenommen, sondern die von der Probe durchgelassene Gesamtintensität in ausgewählten spektralen Bereichen gemessen, welche mittels optischer Bandpässe festgelegt werden. Beispielhaft sind in Abbildung 3 die optischen Durchlassbereiche von vier Bandpassfiltern im Vergleich zu den MIR-ATRAbsorptionsspektren von wässrigen Ethanolund Saccharose-Lösungen dargestellt. Da Ethanol eine spezifische Absorptionsbande bei
$2980 \mathrm{~cm}^{-1}$ besitzt, wurde für dessen quantitative Bestimmung ein Filter gewählt, welches in diesem Spektralbereich eine hohe Transmission aufweist und außerhalb dieses Bereichs blockiert. Für die Messung von Zucker in Getränken wurde ein Filter mit hohem Durchlassgrad im Bereich von $1135-1165 \mathrm{~cm}^{-1}$ gewählt, weil hier die Kohlenhydrate Glucose, Fructose und Saccharose annähernd gleich stark absorbieren. Für die Ethanol- und die Zuckerbestimmung wurden Referenzkanäle in spektraler Nachbarschaft zum jeweiligen Messkanal verwendet, um die Absorption der Probenmatrix und temporäre Drifteffekte des optischen Aufbaus möglichst gut zu kompensieren. Mit diesen vier optischen Bandpassfiltern wurde der Detektor im Prototyp des MIR-ATR-Sensors bestückt.

\section{Anwendung in der Getränkeanalyse}

Die Kalibration für die Bestimmung von Zucker und Ethanol in Getränken erfolgte anhand der Zweistoffgemische Saccharose/Wasser und Ethanol/Wasser sowie des Dreistoffgemischs Ethanol/Saccharose/Wasser. In Abbildung 4 ist zu erkennen, dass die beiden Analyten Ethanol und Saccharose im Zweistoffgemisch mit einer Wiederfindungsrate nahe $100 \%$ bestimmt werden können. Dagegen weist insbesondere die Saccharose-Bestimmung im Dreistoffgemisch einen Fehler von bis zu 15\% auf.

Tab. 1: Vergleich der mit dem MIR-ATR-Sensor gemessenen Ethanol- und Zuckerkonzentrationen mit den Herstellerangaben.

\begin{tabular}{|c|c|c|}
\hline & $\begin{array}{c}\text { Angabe } \\
\text { (Sollwert) }\end{array}$ & Messung \\
\hline Ethanol in Bier & $4,8 \mathrm{Vol} . \%$ & $5,0 \mathrm{Vol} . \%$ \\
\hline Zucker in Cola & $106 \mathrm{~g} / \mathrm{L}$ & $117 \mathrm{~g} / \mathrm{L}$ \\
\hline Ethanol im Gemisch & $2,4 \mathrm{Vol} . \%$ & $2,6 \mathrm{Vol} . \%$ \\
\hline Ethanol in Cola & $0 \mathrm{Vol} . \%$ & $0,2 \mathrm{Vol} . \%$ \\
\hline $\begin{array}{c}\text { Zucker in Bier } \\
\text { (siehe Text) }\end{array}$ & k.A. & $51 \mathrm{~g} / \mathrm{L}$ \\
\hline Zucker im Gemisch & k.A. & $91 \mathrm{~g} / \mathrm{L}$ \\
\hline
\end{tabular}

Nach erfolgter Kalibration wurden mit dem Sensor ein Bier (Alkoholgehalt 4,8 Vol.\%), eine Cola (Zuckergehalt $106 \mathrm{~g} / \mathrm{L}$ ) und ein 1:1Gemisch aus beiden Getränken analysiert. Anhand der Tabelle 1 ist zu erkennen, dass der ermittelte Alkoholgehalt im Bier um 0,2 Vol. \% (relative Abweichung ca. $+4 \%$ ) und der Zuckergehalt um $11 \mathrm{~g} / \mathrm{L}$ (relative Abweichung $+10 \%$ ) über den jeweiligen Herstellerangaben liegen. In der 1:1-Mischung wurden 2,6 Vol.\% Ethanol gefunden (Sollwert 2,4 Vol.\%). Die Zuckermenge im Bier wurde zu $51 \mathrm{~g} / \mathrm{L}$ ermittelt. 
Da sich auf dem Etikett keine Angaben zu den im Bier enthaltenen Zuckern (überwiegend nicht einkalibrierte Dextrine) befanden, konnte dieser Wert nicht verifiziert werden. Damit der MIRATR-Sensor die derzeit in der Getränke- industrie etablierten Messgeräte wie Refraktometer und Dichteschwinger ersetzen kann, muss jedoch der Messfehler für Alkohol deutlich unter $0,1 \mathrm{Vol} \% \%$ liegen.

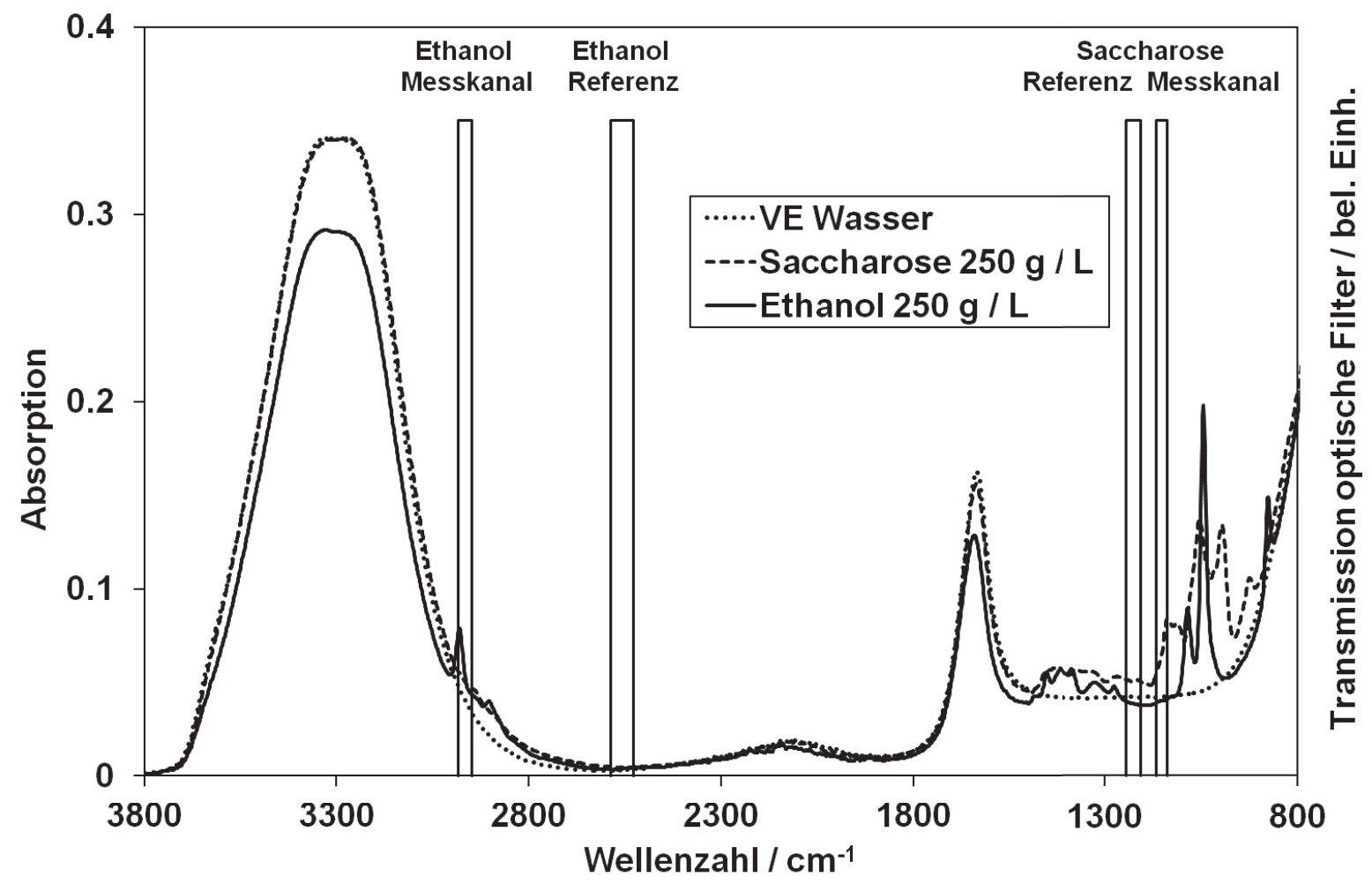

Abb. 3: Transmission der eingesetzten optischen Bandpassfilter und MIR-ATR-Spektren von wässrigen Ethanolund Saccharose-Lösungen.

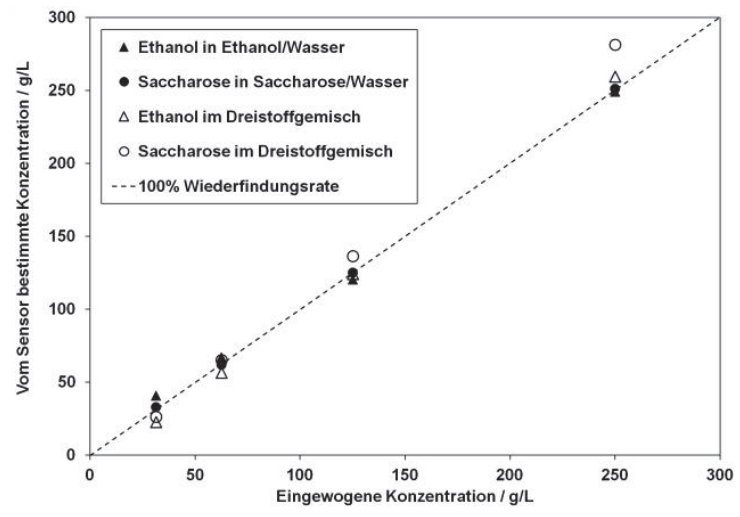

Abb. 4: Vom MIR-ATR-Sensor gemessene Konzentration von Ethanol und Saccharose in wässrigen Lösungen im Vergleich zur Einwaage bei der Probenpräparation.

\section{Anwendung in der Prozesskontrolle}

Der Prototyp des MIR-ATR-Sensors war zunächst für den Einsatz in der Getränkeindustrie mit optischen Bandpassfiltern für die
Zucker- und Ethanolbestimmung ausgestattet worden. Daher wurde der Sensor bei einem Prozess erprobt, dessen Verlauf mit einer Messung dieser beiden Analyten beurteilt werden kann. Bei einer Hefe-Fermentation wird das Substrat Zucker (Saccharose) abgebaut und das Produkt Ethanol im Laufe der Zeit gebildet. Während einer neunstündigen HefeFermentation wurden im Abstand von einer Stunde Proben entnommen und diese sowohl mit dem ATR-MIR-Sensor als auch mit einem kommerziellen MIR-Spektrometer (Gerät Alpha mit ATR-Modul, Fa. Bruker Optik $\mathrm{GmbH}$ ) vermessen. In Abbildung 5 sind die mit den MIR-ATR-Messungen ermittelten zeitlichen Verläufe der Zucker- und Ethanolkonzentration im Vergleich zur Referenzanalytik [6] dargestellt. Beim Abbau des Substrats Zucker lieferten der MIR-ATR-Sensor und das MIRATR-Spektrometer vergleichbare Werte. Dabei wurde bei der Zucker-Kalibration des MIR-ATRSpektrometers derselbe Spektralbereich wie beim MIR-ATR-Sensor verwendet. Allerdings 
konnte Ethanol nicht im Messkanal um $2980 \mathrm{~cm}^{-1}$ bestimmt werden, da hier eine hohe Querempfindlichkeit zu anderen Substanzen vorlag. Die Ursache hierfür ist die komplexe Zusammensetzung der Zellsuspension im Fermenter. Im Fall des MIR-ATR-Spektrometers wurde deshalb die Ethanolbestimmung in einem anderen Spektralbereich um $1050 \mathrm{~cm}^{-1}$ durchgeführt. Der damit ermittelte Verlauf der Ethanolkonzentration zeigt eine gute Übereinstimmung mit der Referenzmethode. Bei einer Bestückung des MIR-ATRSensors mit entsprechenden optischen Bandpassfiltern sollte dieser analog zur Zuckerbestimmung die gleichen Messwerte wie das MIR-ATR-Spektrometer liefern.

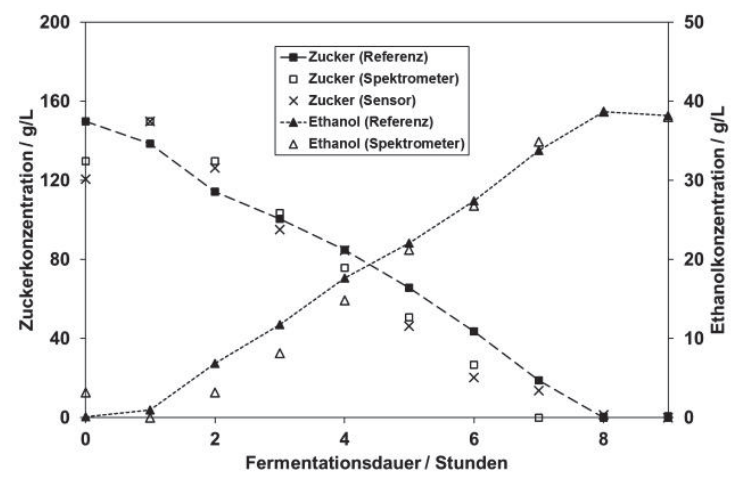

Abb. 5: Zeitliche Verfolgung des Zuckerabbaus und der Ethanolproduktion bei einer aeroben HefeFermentation mittels photometrischem MIR-ATRSensor (Zucker) und MIR-ATR-Spektrometer (Zucker, Ethanol).

\section{Ausblick}

Der Prototyp des MIR-ATR-Sensors wurde mit optischen Bandpassfiltern für die photometrische Messung von Ethanol und Zucker in wässrigen Proben (Getränke, HefeFermentation) ausgestattet. Der Sensor kann durch Filtertausch einfach auf andere biotechnologische, chemische und pharmazeutische Prozesse umgerüstet werden. Es ist geplant, den Sensor in der Chemieindustrie bei einem großtechnischen Verfahren zu erproben. Allerdings stellen hier Randbedingungen wie Druck- und Temperaturstabilität sowie chemische Resistenz eine Herausforderung dar. Bei dieser Anwendung ist es jedoch von Vorteil, dass die Edukte und Produkte häufig in einer höheren Konzentration vorliegen als bei der aufgeführten Hefe-Fermentation. Mit der derzeit erreichten Langzeitstabilität des MIRATR-Sensors sollte daher eine Abbildung des chemischen Reaktionsverlaufs gut möglich sein.

\section{References}

[1] G. Mazarevica, L. Diewok, J.R. Baena, E. Rosenberg and E. Lendl, On-Line Fermentation Monitoring by Mid-infrared Spectroscopy, Appl. Spectrosc. 58(7), 804-810 (2004)

[2] S. Sivakesava, J. Irudayaraj and A. Demirci, Monitoring a bioprocess for ethanol production using FT-MIR and FT-Raman spectroscopy, J. Ind. Microbiol. Biot. 26, 185-190 (2001)

[3] P. Roychoudhury, L.M. Harvey and B. McNeil, The potential of mid infrared spectroscopy (MIRS) for real time bioprocess monitoring, Anal. Chim. Acta. 571, 159-166 (2006)

[4] M. Otto, Analytische Chemie, Wiley-VCH, ISBN 3-527-29840-1 (2000)

[5] N.J. Harrick, Internal Reflection Spectroscopy, John Wiley \& Sons Inc, ISBN 0470352507 (1967)

[6] T. Beuermann, D. Egly, D. Geoerg, K.I. Klug, W. Storhas and F.J. Methner, On-line carbon balance of yeast fermentations using miniaturized optical sensors, J. Biosci. Bioeng. 113(3), 399405 (2012) 